\title{
MRI measurement on intercondylar notch after anterior cruciate ligament rupture and its correlation
}

\author{
XIAO OUYANG ${ }^{1}$, YU HAO WANG ${ }^{2}$, JIAN WANG $^{3}$, SHI DONG HONG $^{1}$, FENG XIN $^{1}$, LIN WANG $^{1}$, \\ XIAO WEI YANG ${ }^{1}$, JING RONG WANG $^{1}$, LI MING WANG ${ }^{4}$, BO WEI $^{4}$, \\ QING WANG ${ }^{5}$, WEI DING CUI ${ }^{5}$ and XING LI FU ${ }^{6}$
}

\begin{abstract}
${ }^{1}$ Department of Orthopedics, Xuzhou Third Hospital, Affiliated Hospital of Jiangsu University, Xuzhou, Jiangsu 221005;
${ }^{2}$ Nanjing Medical University, Nanjing, Jiangsu 211166; ${ }^{3}$ Hospital Administration Office, Xuzhou Third Hospital, Affiliated Hospital of Jiangsu University, Xuzhou, Jiangsu 221005; ${ }^{4}$ Department of Orthopedics, Nanjing First Hospital, Affiliated Hospital of Nanjing Medical University, Nanjing, Jiangsu 210006; ${ }^{5}$ Department of Orthopedics,

The First Affiliated Hospital of Nanjing Medical University, Nanjing, Jiangsu 210029;

${ }^{6}$ Jiangsu University Health Science Center, Zhenjiang, Jiangsu 212001, P.R. China
\end{abstract}

Received November 25, 2015; Accepted February 16, 2016

DOI: $10.3892 / \mathrm{etm} .2016 .3078$

\begin{abstract}
The knee joint is extremely susceptible to injury, which is usually identified by magnetic resonance imaging (MRI). In the present study, MRI was applied to quantitatively detect the association between anterior cruciate ligament (ACL) rupture and anatomic morphologic changes of the intercondylar notch. Forty patients with unilateral ACL rupture who were treated between July, 2013 and October, 2014 were enrolled in the present study. The patients were divided into the observation (affected side) and control (healthy side) groups. MRI measurements were undertaken based on parameters associated with intercondylar notch of double knee joints. The results showed that intercondylar notch width (ICW) in the observation group was significantly smaller than that in the control group, and differences were statistically significant $(\mathrm{P}<0.05)$. Differences on the intercondylar notch height and femoral condyle width [epicondylar width (EW)] between the two groups were not statistically significant $(\mathrm{P}>0.05)$. Notch width index (NWI) and notch shape index (NSI) in the observation group were significantly less than those in the control group and differences were statistically significant $(\mathrm{P}<0.05)$. Differences of Lysholm and Tegner scoring between the two groups were not statistically significant $(\mathrm{P}>0.05)$. The differential value of ICW in the observation group was
\end{abstract}

Correspondence to: Dr Xiao Ouyang, Department of Orthopedics, Xuzhou Third Hospital, Affiliated Hospital of Jiangsu University, 131 Huancheng Road, Xuzhou, Jiangsu 221005, P.R. China

E-mail: ouyangxiaoxz@sina.com

Dr Xing Li Fu, Jiangsu University Health Science Center, 3 Yizheng Road, Zhenjiang, Jiangsu 212001, P.R. China

E-mail:xingli_fu1@163.com

Key words: cruciate ligament rupture, femoral intercondylar notch, magnetic mesonance imaging, Lysholm scoring, Tegner scoring
$2.6 \pm 1.3 \mathrm{~mm}$ and the ACL rupture time of the affected knee was $20.4 \pm 1.3$ months on average. The correlation was statistically significant $(\mathrm{P}<0.05)$. The correlation of Lysholm scoring, Tegner scoring and intercondylar notch stenosis degree on the affected knee was not statistically significant $(\mathrm{P}>0.05)$. In conclusion, after ACL rupture, ICW on the affected knee had significant stenosis, NSI and NWI were significantly reduced and the stenosis degree was aggravated with the prolongation of course. By contrast, Lysholm and Tegner scoring of patients with different degrees of stenosis had no correlation.

\section{Introduction}

Knee joint is one of the most vulnerable joints in the human body. Its ligamentous injury incidence is relatively high, at $15 \%$. Due to its unique anatomical structure and anatomical effect, the anterior cruciate ligament (ACL) is extremely vulnerable to injuries, accounting for approximately $56 \%$ of all knee joint ligaments (1). Femoral intercondylar notch is an important anatomical structure in the distal part of femur. ACL injury is closely associated with the size of the femoral intercondylar notch, considered susceptible to ACL injury. Therefore, it is critical to investigate the femoral intercondylar notch (2).

Magnetic mesonance imaging (MRI) has a good soft tissue imaging ability as well as a high soft tissue resolution and differentiates various results precisely. Thus it is beneficial in clinic application (3). In the present study, we studied the association between ACL rupture and anatomic morphologic changes of the intercondylar notch and analyzed the correlation between the course of ACL and knee joint motor function scoring, thus providing a reference for clinical treatments of ACL rupture patients.

\section{Materials and methods}

General materials. Forty patients with unilateral ACL rupture treated at the Xuzhou Third Hospital (Jiangsu, China) 
between July, 2013 and October, 2014, were enrolled in the current study. The patients were from multiple professions and were confirmed without disease history on the knee joint. Of the 40 cases, 22 cases were men and 18 were women, aged 20-62 years, with an average of $32.2 \pm 2.3$ years. The patients had a disease history of 2-28 months, with an average of $20.4 \pm 1.3$ months. Eighteen cases of ACL were identified on the left knee while 23 cases were on the right knee.

Approval for the present study was obtained from the Ethics Committee of Xuzhou Third Hospital. Informed consent was obtained from the patients.

The inclusion criteria for the study were: i) patients $\geq 18$ years old; ii) patients whose affected knee joints had different degrees of pain after movement, limited leg extension, knee joint effusion or knee joint instability as well as other clinical performances; iii) patients who accepted X-ray and nuclear MRI (NMRI) examination on the affected side prior to treatment and had complete materials; iv) patients who had no significant degenerative changes on the affected knee joint under X-ray examination; v) patients whose injury conditions on cruciate ligaments, meniscus and cartilage on articular surface were confirmed under the detection of laparoscopic surgery and who accepted corresponding treatments on injury condition. The exclusion criteria for the study were: i) patients with direct contact injuries or high-energy violence injuries, such as car accident and falling accidents; ii) patients with bilateral knee joint injuries or whose affected knee joint had multiple ligament injury; iii) patients with uneven density on the intercondylar notch and articular surface under X-ray examination, with condyle osteophyte formation on median and lateral femur as well as on tibial intercondylar eminence or with obvious degeneration of the knee joint; iv) patients with a past history of knee joint tuberculosis, suppurative arthritis, rheumatism or rheumatoid arthritis and other knee joint diseases; v) patients with knee joint tumor or tumor-like lesion; vi) patients with a past history of trauma or related operation on the affected knee joint; vii) patients with severe hepatic renal dysfunction; viii) patients with poor compliance and patients that declined participation in the study.

Methods. The 40 patients were diagnosed by 4-limb joint image diagnostic apparatus produced by Italian Esaote (Genova, Italy) professional knee joint-phased array surface coil, and the magnetic field intensity was $0.2 \mathrm{~T}$. The scanning sequence performed were as follows: coronal plane spin-echo (SE) sequence T2W1, TR2100 ms and TE90 ms; vertical plane SE sequence T1W1, TE90 ms and TR2100 msl; cross section GE sequence TE18 ms and TR540 ms, with deflecting angle of $40^{\circ}$, a layer thickness of $3.5 \mathrm{~mm}$, FOV 170x170 mm, interlamellar spacing of $0.4 \mathrm{~mm}$. The patients were maintained in a supine position until the lower patella was located in the central part of the coil. The knee joints of the patients were bent to $10^{\circ}$ until the border of the intercondylar notch was adjacent to the anatomic landmark. The trailing edge was the initial image of the trailing edge joint of the femoral condyle, while the leading edge was the terminal image of the medial and lateral condyle which maintained continuity. The trailing edge served as the line of bilateral condylar joint surface.
Observation indications. The patients were divided into the observation group (affected side) and the control group (healthy side). MRI measurements were performed to determine whether on the NMRI coronal plane SE sequence T2W1 image, the border of the intercondylar notch was consistent with the anatomic landmark. The anterior border was the initial clear and continuous image of medial and lateral condyle. The posterior border was the terminal clear image of medial and lateral condyle. The lower border was the line between the lowest points of cartilage surface of the medial and lateral condyle. The initial image showing continuity in the medial and lateral condyle and which showed a clear notch shape and popliteus tendon sulcus of the lateral condyle was considered the standard. Line I was denoted as the line between the lowest points of cartilage surface of medial and lateral condyle. A line parallel to line I on the popliteus tendon sulcus was drawn, and designated as line II. The distance between line II and the crossing point of the medial and lateral walls of the femoral intercondylar notch as intercondylar notch width (ICW) was measured. To determine the distance between line II and the crossing point of the medial condyle and lateral condyle of the femur as EW, a line perpendicular to line I from the highest point of the intercondylar notch was drawn and the height of this line was determined as intercondylar notch height (ICH). The ratio of ICW and EW was the notch as notch width index (NWI), and that of ICW and ICH as the notch shape index (NSI). Lysholm and Tegner scoring on the affected knee joints was performed and the differences of the aforementioned indices were compared. The correlation between ICW and disease progression as well as knee joint motor function scoring was analyzed.

Statistical analysis. The SPSS 18.0 software package (SPSS, Inc., Chicago, IL, USA) was applied to process the data. Measurement data were presented as means \pm standard deviation. The t-test was used to determine comparisons between groups. Enumeration data were presented by case or percentage. The $\chi^{2}$ test was used to determine comparisons between groups. $\mathrm{P}<0.05$ was considered to indicate a statistically significant difference.

\section{Results}

Comparisons of ICW, ICH and EW between the two groups. ICW in the observation group was significantly smaller than that in the control group and differences were statistically significant $(17.3 \pm 2.1 \mathrm{~mm}$ vs. $22.5 \pm 2.6 \mathrm{~mm} ; \mathrm{P}<0.05)$. Differences of ICH in the two groups was not statistically significant $(31.3 \pm 2.6 \mathrm{~mm}$ vs. $30.9 \pm 2.5 \mathrm{~mm}$; P>0.05; Table I).

Comparisons of NWI and NSI between the two groups. NWI and NSI in the observation group were significantly less than those in the control group. Differences were statistically significant (NWI: $0.201 \pm 0.03$ vs. $0.253 \pm 0.04, \mathrm{P}<0.05$; NSI: $0.521 \pm 0.003$ vs. $0.564 \pm 0.005, \mathrm{P}<0.05$; Table II).

Comparisons of knee joint function scoring. Differences of Lysholm and Tegner scoring between the two groups were not statistically significant (Lysholm scoring: $32.3 \pm 7.4$ vs. 34.6 \pm 5.2 , $\mathrm{P}>0.05$; Tegner scoring: $5.2 \pm 1.1$ vs. $5.3 \pm 1.3, \mathrm{P}>0.05$; Table III). 
Table I. Comparisons of ICW, ICH and EW between the two groups (mm).

\begin{tabular}{lccc}
\hline Groups & ICW & ICH & EW \\
\hline Observation & $17.3 \pm 2.1$ & $31.3 \pm 2.6$ & $77.5 \pm 3.8$ \\
Control & $22.5 \pm 2.6$ & $30.9 \pm 2.5$ & $78.2 \pm 3.9$ \\
T & 2.843 & 0.701 & 0.813 \\
P-value & 0.037 & 0.483 & 0.416 \\
\hline
\end{tabular}

ICW, intercondylar notch width; ICH, intercondylar notch height; EW, epicondylar width.

Table II. Comparisons of NWI and NSI between the two groups.

\begin{tabular}{lcc}
\hline Groups & NWI & NSI \\
\hline Observation & $0.201 \pm 0.03$ & $0.521 \pm 0.003$ \\
Control & $0.253 \pm 0.04$ & $0.564 \pm 0.005$ \\
$\mathrm{~T}$ & 2.572 & 2.643 \\
P-value & 0.035 & 0.029 \\
\hline
\end{tabular}

NWI, notch width index; NSI, notch shape index.

Table III. Comparisons of knee joint function scoring.

\begin{tabular}{lcc}
\hline Groups & Lysholm scoring & Tegner scoring \\
\hline Observation & $32.3 \pm 7.4$ & $5.2 \pm 1.1$ \\
Control & $34.6 \pm 5.2$ & $5.3 \pm 1.3$ \\
T & 0.363 & 0.275 \\
P-value & 0.715 & 0.624 \\
\hline
\end{tabular}

Analysis of the correlation between ICW and disease progression as well as knee joint motor function scoring. The differential value of ICW in the observation group was $2.6 \pm 1.3 \mathrm{~mm}$ and the ACL rupture time of the affected knee was $20.4 \pm 1.3$ months on average. The correlation was statistically significant $(r=0.721, \mathrm{P}<0.01$; Fig. 1). Correlation of Lysholm scoring, Tegner scoring and intercondylar notch stenosis degree on the affected knee were not statistically significant $(\mathrm{r}=-0.236, \mathrm{P}>0.05 ; \mathrm{r}=-0.127, \mathrm{P}>0.05)$.

\section{Discussion}

Attention has been focused on the association between ACP and intercondylar notch. The size of intercondylar notch was closely associated with ACP rupture (2). Patients with narrow intercondylar notch are considered more susceptible to ACP injury. Major factors resulting in the narrowness of intercondylar notch included joint degeneration, congenital malformation, long-term instability of the knee joint and reconstruction failure of the ruptured ACL. The lump between ACL and femoral intercondylar notch resulted in limited straightening of the knee joint, pain and other symptoms (4).

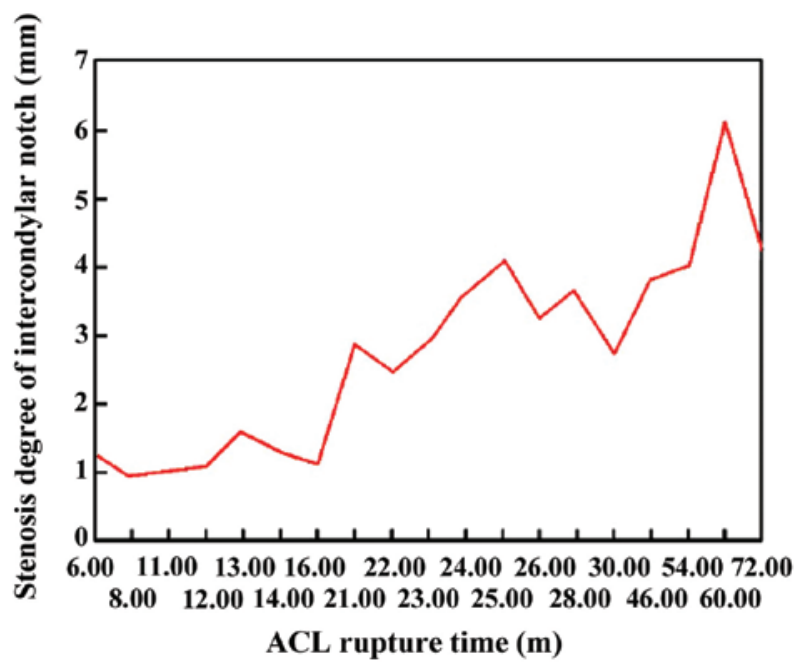

Figure 1. Association between stenosis degree of intercondylar notch and rupture time in the observation group. ACL, anterior cruciate ligament.

Therefore, investigation of intercondylar notch and an analysis of their effects in the treatment of patients with ACL rupture is crucial. MRI is commonly used in the detection of orthopedic trauma and soft tissue lesions. Different sequences of MRI have different application values in varying tissue signals and quantitatively detect relevant anatomical structures (5).

The results of the present study have shown that ICW in the observation group was significantly smaller than that in the control group and differences were statistically significant. Differences of ICH and EW between the two groups were not statistically significant. NWI and NSI in the observation group were significantly less than those in the control group and differences were statistically significant. By contrast, differences of the Lysholm and Tegner scoring between the two groups were not statistically significant. Additionally, the differential value of ICW in the observation group and ACL rupture time of the affected knee was positively correlated. By contrast, the correlation between Lysholm scoring, Tegner scoring, and intercondylar notch stenosis degree on the affected knee was not statistically significant. NSI served as the ratio between ICW and ICH. The greater the ratio, the more rounded the intercondylar notch. While extending their knee joints, ACL on patients with a relatively low ratio would become tight and most of the ligaments would be in the anterior part of intercondylar notch. Thus, the narrow intercondylar notch did not provide sufficient space, resulting in dysfunctions of ACL (6). The greater the NSI, the more likely the intercondylar notch was able to provide sufficient space for ACL, when the patients extended their knee joints.

The abovementioned results have confirmed the association between intercondylar notch stenosis and ACL rupture and that intercondylar notch stenosis was one of the key factors resulting in ACL rupture. These results are consistent with those of previous reports (7). However, the literature seldom mentions the association between ICH and ACL rupture. A possible reason is that the measurement criteria of $\mathrm{ICH}$ were uncertain, and due to the measurement having a certain degree of difficulty, exact data could not be obtained (8). The results of the present study have shown that differences of 
ICH and EW between the two groups were not statistically significant. By contrast, the differences of ICW between the two groups were statistically significant, indicating that ICW was important to ACL rupture. Additionally, the shape and width of the femoral intercondylar notch were important factors that affect ACL rupture.

Direct indications of ACL rupture under MRI examination included (9): ACL disappearance or shrinking, discontinuity, abnormal contour, edema and thickening. Indirect indications of ACL rupture included lateral and posterior tibia contusion, femur lateral condyle contusion, kissing bone contusion, lateral meniscus posterior horn exposure, anterior disc displacement of tibia, PCL curvature, posterior PCL line and patellar tendon tortuosity, decrescent angle between ACL and tibial plateau, Segond fracture, trigonum effusion and medial collateral ligament injury (10). Use of MRI in the diagnosis of complete ACL rupture is common with the sensibility and specificity of MRI in diagnosing ACL rupture reaching 89 and $84 \%$, respectively (11). However, there was still a certain degree of false-positive and -negative results. Common reasons for false-positive results included hypertrophic synovium in intercondylar notch, scar healing degeneration of ACL, structural artifacts on tissues and organs between joint capsule and ACL, including tendon, synovial membrane, fat and blood vessel. Common reasons for false-negative results included the separation of integrated end of ligament under incomplete ACL fracture, formation of edema and scar under long-term injury and disappearance of localized high signal $(12,13)$. Therefore, MRI examination prior to arthroscopic surgery is crucial as it may effectively reduce or avoid the implementation of arthroscopic surgery. MRI showed images on sagittal, coronal and transverse sections and does not result in artifacts. In addition, there was no need to use any contrast agents, thus preventing any adverse effects on the body. MRI had a relatively higher display rate of injuries on joint, tendon and ligament in the musculoskeletal system $(14,15)$. In the present study, all 40 patients accepted MRI examination and obtained clear images as well as exact parameters associated with the intercondylar notch.

In conclusion, following ACL rupture, ICW on the affected knee indicated significant stenosis. NSI and NWI were significantly reduced and the stenosis degree was further aggravated with disease progression. No correlation was found for Lysholm and Tegner scoring of patients with different degrees of stenosis. However, additional studies should be conducted using larger samples for the results to be confirmed.

\section{References}

1. Hunter DJ, Niu J, Zhang Y, Totterman S, Tamez J, Dabrowski C, Davies R, Le Graverand MP, Luchi M, Tymofyeyev Y, Beals CR; OAI Investigators: Change in cartilage morphomerty: a sample of the progression cohort of the Osteoarthritis Initiative. Ann Rheum Dis 68: 349-356, 2009.

2. Cha JH, Lee SH, Shin MJ, Choi BK and Bin SI: Relationship between mucoid hypertrophy of the anterior cruciate ligament (ACL) and morphologic change of the intercondylar notch: MRI and arthroscopy correlation. Skeletal Radiol 37: 821-826, 2008.

3. Clayton RA and Court-Brown CM: The epidemiology of musculoskeletal tendinous ligamentous injuries. Injury 39: 1338-1344, 2008.

4. Boden BP, Torg JS, Knowles SB and Hewett TE: Video analysis of anterior cruciate ligament injury: abnormalities of hip and ankle kinematics. Am J Sports Med 37: 252-259, 2009.

5. Amin S, Guermazi A, Lavalley MP, Niu J, Clancy M, Hunter DJ, Grigoryan M and Felson DT: Complete anterior cruciate ligament tear and the risk for cartilage loss and progression of symptoms in men and women with knee osteoarthritis. Osteoarthritis Cartilage 16: 897-902, 2008.

6. Podraza JT and White SC: Effect of knee flexion angle on ground reaction forces, knee moments and muscle co-contraction during an impact-like deceleration landing: implications for the non-contact mechanism of ACL injury. Knee 17: 291-295, 2010.

7. Mizuno K1, Andrish JT, van den Bogert AJ and McLean SG: Gender dimorphic ACL strain in response to combined dynamic 3D knee joint loading: implications for ACL injury risk. Knee 16: 432-440, 2009.

8. Boden BP, Sheehan FT, Torg JS and Hewett TE: Noncontact anterior cruciate ligament injuries: mechanisms and risk factors. J Am Acad Orthop Surg 18: 520-527, 2010.

9. Nagano Y, Ida H, Akai M and Fukubayashi T: Biomechanical characteristics of the knee joint in female athletes during tasks associated with anterior cruciate ligament injury. Knee 16: 153-158, 2009.

10. Shimokochi Y and Shultz SJ: Mechanisms of noncontact anterior cruciate ligament injury. J Athl Train 43: 396-408, 2008.

11. Simon RA, Everhart JS, Nagaraja HN and Chaudhari AM: A case-control study of anterior cruciate ligament volume, tibial plateau slopes and intercondylar notch dimensions in ACL-injured knees. J Biomech 43: 1702-1707, 2010.

12. Stein V, Li L, Guermazi A, Zhang Y, Kent Kwoh C, Eaton CB and Hunter DJ; OAI Investigators: The relation of femoral notch stenosis to $\mathrm{ACl}$ tears in persons with knee osteoarthritis. Osteoarthritis Cartilage 18: 192-199, 2010.

13. Gianotti SM, Marshall SW, Hume PA and Bunt L: Incidence of anterior cruciate ligament injury and other knee ligament injuries: a national population-based study. J Sci Med Sport 12: 622-627, 2009.

14. Alentorn-Geli E, Myer GD, Silvers HJ, Samitier G, Romero D, Lázaro-Haro C and Cugat R: Prevention of non-contact anterior cruciate ligament injuries in soccer players. Part 1: Mechanisms of injury and underlying risk factors. Knee Surg Sports Traumatol Arthrosc 17: 705-729, 2009

15. Ekstrand J, Hägglund M and Waldén M: Injury incidence and injury patterns in professional football: the UEFA injury study. Br J Sports Med 45: 553-558, 2011. 